\title{
COMMUNICATION
}

\section{Traduction CBCT des Ostéodystrophies rénales}

\section{Gossiome $\mathbf{C}^{1}$, Moreau $\mathbf{N}^{1,2}$, Daviet-Noual $\mathbf{V}^{1}$, Chaussain $\mathbf{C}^{1,3}$ Ejeil A.-L.', Salmon B ${ }^{1,3}$}

1- AP-HP - Hôpitaux Universitaires Paris Nord Val de Seine, Services d'Odontologie Bretonneau, Paris, France

2- Labo NM

3- EA 2496, Université Paris Descartes Sorbonne Paris Cité, Faculté de Chirurgie Dentaire, Montrouge, France

\section{Introduction}

L'ostéodystrophie est une complication osseuse, retrouvée lors d'une insuffisance rénale chronique, particulièrement chez les patients dialysés. Elle apparaît lorsque les reins échouent à maintenir une calcémie et une phosphatemie normales. II en résulte une dérégulation du métabolisme phosphocalcique qui se traduit par une hypersécrétion de parathormone. Cette hormone va provoquer la libération de calcium et de phosphate osseux par une stimulation de l'ostéoclasie, perturbant ainsi le remodelage osseux. Les changements de structure dus à l'ostéodystrophie d'origine rénale, conduisent à un os plus fin et plus fragile pouvant provoquer des douleurs osseuses et une susceptibilité accrue aux fractures. Le traitement de l'ostéodystrophie d'origine rénale repose sur une supplémentation en calcium et en vitamine $D$ en attente d'une transplantation rénale.

\section{Observation}

L'objectif est de présenter, à travers une série de cas, la traduction radiologique au niveau dentomaxillo-facial de ces ostéodystrophies chez des patients souffrant d'insuffisance rénale chronique et traités par hémodialyse. L'ostéodystrophie se caractérise par des plages de raréfaction osseuse, qui pourraient être confondues avec des images de résorption pathologique.

Discussion

Les indications du cone beam CT s'élargissent dans la sphère oro-faciale, en particulier en chirurgie orale. Cet examen permet de dépister les lésions osseuses avec une plus grande sensibilité que la radiographie conventionnelle ${ }^{2}$ et les découvertes fortuites sont fréquentes.

Afin d'éviter les erreurs radiodiagnostiques, il est important de connaitre les images évocatrices d'une pathologie systémique avec répercussion sur le métabolisme phosphocalcique, telle que l'ostéodystrophie rénale. Ces situations nous rappellent l'intérêt d'être vigilants lors de l'analyse des images d'un cone beam CT qui doit être complète et intéresser l'ensemble du volume exploré.

\footnotetext{
${ }^{1}$ Vitamin D and chronic kidney disease-mineral bone disease (CKD-MBD). Sagar U nigwekar \& al. BoneKEy Reports 2014 Article ,number 498.

${ }^{2}$ Accuracy of Cone Beam Computed Tomography and Panoramic and Periapical Radiography for Detection of Apical Periodontitis. Carlos Estrela \& al. J Endod 2008 vol 34 273-279
} 